\title{
Die fünf häufigsten Irrtümer über das Ehegattensplitting
}

Das Ehegattensplitting zu verstehen ist nicht einfach. Seine Funktionsweise ist außerhalb des Steuerrechts wenig bekannt. Über die Wirkungen streiten Politik und Wissenschaft schon lange. Streit besteht auch über die Spielräume des Gesetzgebers für alternative Lösungen. Mit der Einführung der eingetragenen Lebenspartnerschaft sind neue Fragen hinzugekommen.

Diese Grauzonen des Wissens zeigen sich in der aktuellen Debatte um die Einführung des Splittings für die eingetragene Lebenspartnerschaft überdeutlich. Der Deutsche Juristinnenbund e.V. (djb) fordert alle an der Debatte beteiligten Akteurinnen und Akteure auf, sich und andere bestmöglich zu informieren. In der folgenden Erklärung ergreift er die Gelegenheit, mit fünf der häufigsten Irrtümer aufzuräumen.

Seit vielen Jahrzehnten plädiert der djb für eine Individualbesteuerung von Eheleuten bei übertragbaren Grundfreibeträgen. „Hieran hält der Verband weiterhin grundsätzlich fest“, erklärt Prof. Dr. Margarete Schuler-Harms, Vorsitzende der Kommission Recht der sozialen Sicherung, Familienlastenausgleich: „Er wird sich an allen Debatten zur Reform der Ehe-, Partnerschafts- und Familienbesteuerung aktiv beteiligen."

\section{Irrtu3m Nr. 1:}

Das Ehegattensplitting schützt generell die Ehe als Lebensgemeinschaft zur Gründung einer Familie.

Richtig ist: Die Ehe steht unter dem besonderen Schutz des Staates (Art. 6 Abs. 1 GG).

Allerdings profitieren vom „Splitting-Vorteil“ keineswegs alle Eheleute. Er wirkt sich nur auf solche Ehen aus, in denen die steuerpflichtigen Eheleute unterschiedlich hohe Jahreseinkommen haben. Das Ehegattensplitting „belohnt“ also den Verzicht eines Ehepartners auf Erwerbstätigkeit oder die Ausübung von Teilzeitarbeit. Es verteuert kalkulatorisch den (Wieder-)Einstieg nach einer Erziehungs- oder Pflegezeit und begünstigt die Aufnahme einer geringfügigen Beschäftigung, da sonst das neu erwirtschaftete Einkommen zu einem hohen Grenzsteuersatz besteuert werden muss.

Das 1958 eingeführte Ehegattensplitting basierte auf der Idee, dass die Ehefrau traditionell nicht erwerbstätig war und der Mann ein Leben lang sein Einkommen mit ihr teilte. Dieses (Leit-)Bild der lebenslang gültigen Versorgerehe hat der Gesetzgeber in anderen Rechtsbereichen längst aufgegeben. Seit der Unterhaltsreform im Jahr 2008 ist jede Ex-Ehefrau und jeder Ex-Ehemann prinzipiell gehalten, für das eigene Einkommen Sorge zu tragen, und zwar auch neben der Erziehung und Betreuung von Kindern. Außerdem erhalten Kinder aus neuen (ehelichen und nichtehelichen) Verbindungen vorrangig vor der ehemaligen Partnerin oder dem ehemaligen Partner Unterhalt, wenn das Geld nicht für alle reicht. Das steuerrechtliche Bild der Versorgerehe, deren Wirkungen über den Bestand der Ehe hinaus reichen, findet damit im übrigen Recht und in der Lebenswirklichkeit keine Grundlage mehr. Das Festhalten am Ehe- gattensplitting verstärkt vielmehr die Risiken von Frauen im Falle einer Trennung.

Das Ehegattensplitting privilegiert außerdem oftmals die Differenz zwischen den Einkommen von Frauen und Männern und damit auch den gesamtwirtschaftlichen „Gender Pay Gap“.

\section{Irrtum Nr. 2:}

Das Ehegattensplitting kommt vor allem Familien mit Kindern zugute.

Richtig ist, dass heute Ehen mit Kindern in der Summe vom Ehegattensplitting stärker profitieren als Ehen ohne Kinder.

Allerdings profitieren nichteheliche Familien mit Kindern nicht, und zwar selbst dann nicht, wenn sie - wie die eingetragene Lebenspartnerschaft - rechtlich als Solidargemeinschaft ausgestaltet sind. Sie werden allein durch Kinderfreibeträge und Kindergeld entlastet, obwohl die Familie ebenso wie die Ehe unter dem besonderen Schutz des Staates steht. Dabei ergeben sich finanzielle Aufwendungen, für die es einer Entlastung bedarf, typischerweise durch Kinder und nicht (mehr) durch eine Heirat.

Im Lebenslauf eines Kindes enden die finanziellen Wirkungen des Ehegattensplittings, wenn die Eltern sich trennen oder sich scheiden lassen. Hiervon sind heute mehr als ein Drittel, in Großstädten sogar die Hälfte aller Ehen betroffen. Auch Ehen mit Kindern werden mehr und mehr geschieden. Aus Sicht der Kinder zeigt sich dann: Nicht nur die Ausgaben steigen, da anstelle eines Haushalts nun zwei Haushalte unterhalten werden müssen. Auch das verfügbare Einkommen wird geringer, weil die steuerlichen Vorteile des „Splitting“ entfallen, da sie nicht an das Kind, sondern an die Ehe der Eltern gebunden sind. Aus Sicht der Kinder ist das Ehegattensplitting deshalb eine unbeständige, nicht verlässliche Förderungsform.

\section{Irrtum Nr. 3:}

Ein Familiensplitting würde nicht mehr die Ehe, sondern generell die Familie steuerlich privilegieren, und wäre deshalb gerechter.

Richtig ist: Ein Familiensplitting würde alle Formen steuerpflichtiger Haushaltsgemeinschaften von Eltern und Kindern umfassen.

Allerdings wären die finanziellen Vorteile für Familien mit Kindern bei einem Familiensplitting keineswegs so groß, wie es auf den ersten Blick scheinen mag. Die Freibeträge für Kinder sind im deutschen Einkommensteuerrecht so hoch, dass vom Familiensplitting vor allem gut verdienende Familien profitieren würden. Die meisten Familien gingen bei dieser Reform leer aus.

Weiterhin würden, soweit eine Erweiterung des Ehegattensplittings zum Familiensplitting erwogen wird, auch Ehen ohne Kinder vom „Splitting“ profitieren. Die Ehebesteuerung bliebe am Bild der Versorgerehe auf Lebenszeit ausgerichtet, obwohl dieses Bild der gelebten Realität nicht mehr entspricht und auch mit den Wertungen des Eheunterhaltsrechts nicht mehr in Einklang steht. 
Irrtum Nr. 4:

Die gemeinsame Ehebesteuerung und das Ehegattensplitting sind verfassungsrechtlich geboten und somit alternativlos.

Richtig ist: Bei der Besteuerung des Einkommens sind sowohl die steuerliche Leistungsfähigkeit der Steuerpflichtigen als auch der Schutz der Ehe zu beachten.

Allerdings hat der Gesetzgeber Spielraum bei der Neuordnung der Ehe- und Familienbesteuerung. Jedem Menschen steht ein Grundfreibetrag (derzeit in Höhe von jährlich 8.130 Euro) als steuerfreies Existenzminimum zu. Nach ganz überwiegender Auffassung müssen Eheleute ohne Rücksicht auf die Einkommensverteilung in der Ehe zwei Grundfreibeträge geltend machen können.

Der weitere Effekt des Ehegattensplittings jedoch, der „Progressionsvorteil“, ist verfassungsrechtlich nicht gefordert. Der Gesetzgeber ist frei, diesen finanziellen Effekt entfallen zu lassen und das Geld gezielt der Förderung von Familien zuzuwenden.

Der Deutsche Juristinnenbund e.V. (djb) fordert seit langer Zeit eine individuelle Besteuerung von Eheleuten bei Übertragbarkeit der existenzsichernden Grundfreibeträge. Die dadurch frei werdenden Geldmittel sollten in gezielte Dienst- und Geldleistungen zugunsten von Familien mit Kindern fließen.
Irrtum Nr. 5:

Der verfassungsrechtliche Schutz der Ehe rechtfertigt eine Ungleichbehandlung von Ehen und eingetragenen Lebenspartnerschaften bei der Besteuerung.

Richtig ist, dass Artikel 6 Absatz 1 GG nicht die eingetragene Lebenspartnerschaft ohne Kinder schützt.

Allerdings fordern Bundesverfassungsgericht und Europäischer Gerichtshof auch, dass eingetragene Lebenspartnerschaften nicht ohne sachlichen Grund diskriminiert werden dürfen. Eingetragene Lebenspartnerschaften sind in Bezug auf das Güter- und Unterhaltsrecht der Ehe gleichgestellt. Mit Recht hat das Bundesverfassungsgericht daher die Gleichstellung unter anderem im Beamten- und im Erbschaftssteuerrecht gefordert, soweit diese an die güter- und unterhaltsrechtlichen Beziehungen anknüpfen. Aus demselben Grunde sind eingetragene Lebenspartnerschaften auch im Einkommensteuerrecht der Ehe gleichzustellen. Eingetragenen Lebenspartnerschaften müssen deshalb zwei Grundfreibeträge ohne Rücksicht auf die Art der Einkommenserzielung zustehen. Einer Erstreckung des Ehegattensplittings auf die eingetragene Lebenspartnerschaft bedarf es aber nicht. Vielmehr sollte aus den schon genannten Gründen die gemeinsame Besteuerung für die Ehe aufgegeben werden.

\section{Ehegattensplitting - Auf dem Weg in das 21. Jahrhundert? \\ Besprechung der Entscheidung des Bundesverfassungsgerichts zur Ungleichbehandlung der eingetragenen Lebenspartnerschaft im Einkommensteuerrecht vom 7. Mai 2013}

\author{
Dr. Ulrike Spangenberg, Dr. Maria Wersig, \\ Mitglieder der djb-Kommission Recht der sozialen Sicherung, \\ Familienlastenausgleich, Berlin
}

Der 2. Senat des Bundesverfassungsgerichts hat in einer im Juni 2013 veröffentlichten langerwarteten Entscheidung ${ }^{1}$ den Ausschluss eingetragener Lebenspartnerschaften vom einkommensteuerrechtlichen Ehegattensplitting für verfassungswidrig erklärt. Die Verpflichtung zur Gleichbehandlung bestehe rückwirkend seit Schaffung der eingetragenen Lebenspartnerschaft im Jahr 2001. Diese Entscheidung war absehbar, denn beide Senate haben in den vergangenen Jahren in vergleichbaren Fällen (zum Beispiel der 1. Senat 2010 zum Erbschafts- und Schenkungssteuerrecht ${ }^{2}$ und der 2. Senat 2012 zum Verheiratetenzuschlag im Beamtenrecht $^{3}$ und zum Grunderwerbsteuerrecht ${ }^{4}$ ) unter Bezug auf den Diskriminierungsschutz in Art. 3 Abs. 3 GG denkbare Rechtfertigungen von Ungleichbehandlungen beider Rechtsinstitute verworfen. Die Begründungsmesslatte für eine Beschränkung der Zusammenveranlagung mit Splittingvorteil auf Ehepaare war inzwischen also außerordentlich hoch. Die Bundesregierung hat bereits rechtzeitig vor Ende der Legislaturperiode ein Gesetz auf den Weg gebracht, wonach das Ehegattensplitting nun auch auf eingetragene Lebenspartnerschaften anwendbar sein soll (BT-Drs. 17/13870). So richtig die Entscheidung in Bezug auf die Anerkennung der eingetragenen Lebenspartnerschaft als der Ehe gleichwertige Institution und „taugliche Grundlage einer Familie“ (Rz. 91) ist. Die Entscheidung hätte Anlass sein können, neu über eine Reform des aus Gleichstellungsgründen kritikwürdigen Ehegattensplittings zu diskutieren. Leider hat der 2. Senat in dieser Hinsicht aber keine klaren Worte zum Ehegattensplitting gefunden, sondern mit Äußerungen zu familienpolitischen Intentionen des Splittings allenfalls neue Verwirrung gestiftet. Aus gleichstellungspolitischer Sicht lässt die Entscheidung wichtige Argumente unbeachtet und bereitet allenfalls den Nährboden für die Einführung eines ebenfalls problematischen Familiensplittings. Der Übergang zur Individualbesteuerung, wie der Deutsche Juristinnenbund sie vorschlägt, wird durch die Entscheidung jedenfalls nicht befördert.

1 BVerfG vom 07.05.2013 - 2 BvR 909/06, 2 BvR 1981/06, 2 BvR 288/07, http://www.bundesverfassungsgericht.de/ entscheidungen/rs20130507_2bvr090906.html (letzter Zugriff: 24.06.2013).

2 BVerfG vom 21.07.2010 - 1 BvR 611/07, 1 BvR 2464/07, BVerfGE 126, 400-433.

3 BVerfG vom 19.06.2012 - 2 BVR 1397/09, NJW 2012, 2790.

4 BVerfC vom 19.06.2012 - 1 BvL 16/11. 